\title{
Checkerboards, Lipschitz functions and uniform rectifiability
}

\section{Peter W. Jones, Nets Hawk Katz and Ana Vargas}

\section{Introduction.}

In his recent lecture at the International Congress [S], Stephen Semmes stated the following conjecture for which we provide a proof.

Theorem 1.1. Suppose $\Omega$ is a bounded open set in $\mathbb{R}^{n}$ with $n>2$, and suppose that $B(0,1) \subset \Omega, \mathcal{H}^{n-1}(\partial \Omega)=M<\infty$. Then there are $\varepsilon>0, L<\infty$ (depending on $n$ and $M$ ) and a Lipschitz graph $\Gamma$ (with constant $L)$ such that $\mathcal{H}^{n-1}(\Gamma \cap \partial \Omega) \geq \varepsilon$.

Here $\mathcal{H}^{k}$ denotes $k$-dimensional Hausdorff measure and $B(0,1)$ the unit ball in $\mathbb{R}^{n}$. By iterating our proof we obtain a slightly stronger result which allows us to cover most of the unit sphere $S^{n-1}$.

Theorem 1.2. Same hypotheses. Given $\delta>0$, there exist $\Gamma_{1}, \ldots, \Gamma_{N}$, $N=N(\delta, M, n)$ so that each $\Gamma_{j}$ is a $C(\delta, M, n)$ Lipschitz graph and

$$
\mathcal{H}^{n-1}\left(\pi\left(\bigcup_{j=1}^{N} \Gamma_{j} \cup \partial \Omega\right)\right) \geq \omega_{n}-\delta,
$$

where $\pi$ denotes the radial projection on $S^{n-1}$ and $\omega_{n}$ is the area of $S^{n-1}$. 
We remark that Theorems 1.1 and 1.2 are somewhat related to the results of $[\mathrm{J}]$. David and Semmes have reported to us [DS2] that they also have proofs of the above theorems. The methods they use are, however, quite different from those we present. Whereas David and Semmes work directly on the domain, we prove a theorem that allows us to stitch together 2-dimensional slices (where the result is trivial). This result, which we call a Checkerboard Theorem, is perhaps the most interesting result of this paper.

Let $[0,1]^{n}$ be the unit cube in $\mathbb{R}^{n}$, and let $A, B \subset \mathbb{R}^{n}$ be Lebesgue measurable sets. We say that $A$ is checkerboard connected through $B$ if for any two points $x, y \in A$, there is a path from $x$ to $y$ which is a finite union of line segments, each line segment in one of the $(n)$-coordinate directions and having both endpoints in $B$. We define $d_{c h, B}(x, y)$, the checkerboard distance to be the infimum over the lengths of such paths. For example, if $A \subset[0,1]^{2}$ is any set and $B=[0,1]^{2}$, then for $x, y=$ $\left(x_{1}, x_{2}\right),\left(y_{1}, y_{2}\right) \in A$ we have

$$
d_{c h, B}(x, y)=\left|x_{1}-y_{1}\right|+\left|x_{2}-y_{2}\right|
$$

On the other hand if $A=B=[0,1 / 3]^{2} \cup[2 / 3,1]^{2}$ then the points $(0,0),(1,1) \in A$ are not checkerboard connected through $B$.

Theorem 1.3. (The Checkerboard Theorem) Given any $\delta>0$ and any measurable set $B \subset[0,1]^{n}$ with $|B|=\varepsilon$, there exists a subset $A \subset B$ with

$$
|A| \geq(1-\delta) \varepsilon^{n}
$$

and with $A$ checkerboard connected through B. Furthermore, there exists a constant $C=C(\delta, \varepsilon, n)<\infty$ such that for all $x, y \in A$,

$$
d_{c h, B}(x, y) \leq C|x-y| .
$$

If $|B|=1-\alpha$ with $\alpha \ll 1$, we can choose $|A| \geq 1-c \alpha$ and $d_{\text {ch }, B}(x, y) \leq$ $\sqrt{n}|x-y|$, for $x, y \in A$.

We remark that the final assertion of Theorem 1.3 provides an approach to a version of Almgren's Tilt Excess Theorem [A]. In that case one has $\varepsilon \sim 1,|B|=1-\alpha$, and one obtains a subset $A$ with $|A| \geq$ $1-C \alpha$ and a Lipschitz mapping $F$ with Lipschitz constant $\leq C \sqrt{n}$. This will be explained more precisely in Section 7 .

An examination of the constants in Theorem 1.3 will allow us to conclude the following 
Corollary 1.4. Suppose $B \subset[0,1]^{n},|B|=\varepsilon$, and $F: B \rightarrow X$ (any metric space) satisfies a Lipschitz condition on any line parallel to the coordinate axes

$$
\rho\left(F\left(x_{1}, \ldots, t, \ldots, x_{n}\right), F\left(x_{1}, \ldots, s, \ldots, x_{n}\right)\right) \leq|t-s|
$$

for any two points on $E$ differing only in one of the $n$ coordinates. Then if $\delta \geq 0$ there exists $A \subset B$ with

$$
|A| \geq(1-\delta) \varepsilon^{n},
$$

and such that $F$ is Lipschitz on $A$,

$$
\rho(F(x), F(y)) \leq C(\delta)\left(n^{3 / 2} \varepsilon^{-n}+\sqrt{n} n^{2 n} \varepsilon^{1-2 n}\right)|x-y| .
$$

The outline of this paper is as follows. In Section 2, we recall a proof of Theorem 1.1 in $\mathbb{R}^{2}$-this a known result included only for the sake of completeness. Section 3 is devoted to the proof of the Checkerboard Theorem. We then check constants to derive Corollary 1.4. This allows us to use the results of Section 2 to derive Theorem 1.1. In Section 4 we provide a counter-example for the checkerboard constant in Theorem 1.3 (equivalently for the Lipschitz constant of Corollary 1.4), showing it must be at least $(\log (1 / \varepsilon))^{1 / 2}(\log \log (1 / \varepsilon))^{-1 / 2}$.

In Section 5, we give another application of our methods by showing how to use two dimensional slices to obtain part of the "Structure Theorem" of geometric measure theory for sets of codimension 1 . In Section 6, we discuss Almgren's Tilt Excess Theorem and the easy case of Theorem 1.3 (i.e. the case when $|B| \sim 1$ ).

\section{A trivial result in $\mathbb{R}^{2}$.}

Let $D_{1}$ denote the closed unit disk in $\mathbb{R}^{2}$. By a radial Lipschitz graph we shall mean a set in $\mathbb{R}^{2}$ given in polar coordinates by the equation $r=f(\theta)$ where $f$ is a $2 \pi$-periodic Lipschitz function. We also define the map $\pi: \mathbb{R}^{2} \backslash 0 \rightarrow S^{1}$ to be radial projection. In this section, we prove

Proposition 2.1. Let $\alpha: S^{1} \rightarrow \mathbb{R}^{2}$ be a closed curve in $\mathbb{R}^{2} \backslash D_{1}$ with degree 1 about 0 . Suppose $\mathcal{H}_{1}\left(\alpha\left(S^{1}\right)\right)<M$, for some $M<\infty$. Then for 
any $\varepsilon>0$ there exists $\Gamma$ a radial Lipschitz graph over $S^{1}$ with Lipschitz constant $C(\varepsilon, M)$ so that

$$
\mathcal{H}_{1}\left(\pi\left(\Gamma \cap \alpha\left(S^{1}\right)\right)\right)>2 \pi-\varepsilon .
$$

Proof. The idea of the proof is that first we prune $\alpha\left(S^{1}\right)$ into a graph and then we trim it to make it Lipschitz . The first observation is that $\nu=\pi(\alpha): S^{1} \longrightarrow S^{1}$ is a well defined continuous map with degree 1 . We may lift it by the universal cover to

$$
\tilde{\nu}: \mathbb{R} \longrightarrow \mathbb{R}
$$

with $\tilde{\nu}(0)=0$ and $\tilde{\nu}(2 \pi)=2 \pi$. Furthermore, since the length of $\alpha$ is bounded, we have that $\tilde{\nu}^{\prime}$ is a signed measure on $[0,2 \pi]$ with total measure $2 \pi$. Define $\rho_{\varepsilon}=\tilde{\nu}^{\prime}-\varepsilon /(4 \pi)$. This is a signed measure on $[0,2 \pi]$ with total measure $2 \pi-\varepsilon /(4 \pi)>0$. We shall now modify $\alpha$ into a curve $\beta$ in such a way that we change only pieces that give rise to sets of measure 0 under $\rho_{\varepsilon}$ and replace them by line segments. Let $L=\sup \left|\tilde{\nu}^{\prime}(I)\right|$ the sup taken over open intervals $I$ in $[0,2 \pi]$ satisfying $\rho_{\varepsilon}(I)=0$. We define $\beta_{1}:[0,2 \pi] \longrightarrow \mathbb{R}^{2}$ to be equal to $\alpha$ except on an interval $I$ with $\rho_{\varepsilon}(I)=0$ and with $\left|\tilde{\nu}^{\prime}(I)\right|>L / 2$. Let $x$ and $y$ be the endpoints of $I$. Let $\beta_{1}(I)$ be the line segment between $\alpha(x)$ and $\alpha(y)$ parametrized so that $\pi\left(\beta_{1}\right)$ has constant speed. We define $\rho_{\varepsilon, 1}$ as before, noting that $\rho_{\varepsilon, 1}(I)=0$. If the measure $\rho_{\varepsilon, 1}$ is nonnegative then $\beta_{1}$ is a graph. Otherwise, $\rho_{\varepsilon, 1}$ is a signed measure with total measure strictly less than that of $\rho_{\varepsilon}$ so that we may proceed recursively, removing intervals with measure $\rho_{\varepsilon, j}$ measure 0 having large total measure and replacing their images with line segments. At last, we obtain $\beta_{\infty}$ whose image is a graph, since associated to it is $\rho_{\varepsilon, \infty}$ which is nonnegative.

We define $\Gamma_{0}=\beta_{\infty}\left(S^{1}\right)$. The next observation is that $\mu=$ $\pi_{*} \mathcal{H}_{1}\left(\Gamma_{0}\right)$ is a well defined positive measure on $S^{1}$ and that,

$$
\int_{S^{1}} \mu \leq M
$$

Let $\mathcal{M}(\mu)$ be the Hardy-Littlewood maximal function of $\mu$. We choose $C$ so that $|\{M(\mu)>C / 2\}|<\varepsilon / 2$. The set $\{M(\mu)>C / 2\}$ is open, hence a union of open intervals and we define the graph $\Gamma$ by replacing the part of $\Gamma_{0}$ over each of these intervals by a line segment between the images of the endpoints. The result is the desired graph with Lipschitz constant $C$. 
By the results of $[\mathrm{J}]$, one can in fact exhaust the image of $\alpha$ by a finite collection of Lipschitz graphs with universal Lipschitz constants and a garbage set with small Hausdorff content.

\section{The checkerboard Theorem.}

Proof of Theorem 1.3. Let $\mathcal{M}_{j}$ be the one dimensional HardyLittlewood maximal operator in the $j$-th coordinate direction. For any set $A$, let $\chi_{A}$ denote its characteristic function and let $A^{c}$ denote $[-1,2]^{n} \backslash A$, i.e. the complement of $A$ in the triple of the unit cube. Choose a small $\alpha>0$ and define

$$
B_{1}=\left\{x \in B: \mathcal{M}_{1}\left(\chi_{B^{c}}\right)(x)<1-\alpha \varepsilon\right\}
$$

and recursively for $j \leq n$,

$$
B_{j}=\left\{x \in B_{j-1}: \mathcal{M}_{j}\left(\chi_{B_{j-1}^{c}}\right)(x)<1-\alpha \varepsilon\right\} .
$$

By choosing $\alpha$ sufficiently small, we may ensure that

$$
\left|B_{n}\right| \geq\left(1-\frac{\delta}{2}\right)^{1 / n} \varepsilon
$$

(This follows easily from the Besicovitch covering Lemma, see [G, p. 39]) In fact, we may choose $\alpha \geq C(\delta) / n^{2}$. We shall now divide up $B_{n}$ into its checkerboard connected components and choose one that suits our purposes. We shall do the same at each scale until we arrive at a set which satisfies a dyadic version of the theorem. A similar treatment as we have just given $B$ will produce the desired set.

For any point in $x \in B_{n}$, we define its good set $\mathcal{G}(x)$ so that $y \in \mathcal{G}(x)$ provided $y \in B$ and there exist $z_{j} \in B_{j}$ when $1 \leq j \leq$ $n-1$ so that $\pi_{1}(y)=\pi_{1}\left(z_{1}\right)$ and so that $\pi_{j}\left(z_{j-1}\right)=\pi_{j}\left(z_{j}\right)$ whenever $2 \leq j \leq n-1$, and so that $\pi_{n}(x)=\pi_{n}\left(z_{n-1}\right)$. Here the $\pi_{j}$ 's are the $(n-1)$-dimensional projections into all but the $j$-th coordinate. In particular for any $y \in \mathcal{G}(x)$ we have that $d_{c h, B}(x, y) \leq 2 n$. Further, by the definition of $B_{n}$, we have that $|\mathcal{G}(x)| \geq \alpha^{n} \varepsilon^{n}$. We define the neighborhod of $x$ to be

$$
N(x)=\left\{y \in B_{n}: \mathcal{G}(x) \cap \mathcal{G}(y) \neq \varnothing\right\}
$$


We cover $B_{n}$ by neighborhoods $N\left(x_{1}\right), \ldots, N\left(x_{M}\right)$ so that for $i \neq j$ we always have $x_{j} \notin N\left(x_{i}\right)$. Then we see that $M<1 /\left(\alpha^{n-1} \varepsilon^{n-1}\right)$ since the $\pi_{1}\left(\mathcal{G}\left(x_{i}\right)\right)$ 's are disjoint and have total measure 1 . Thus, in particular,

$$
M \leq \frac{C(\delta)}{n^{2 n-1}} .
$$

The checkerboard connected components of $B_{n}$ through $B$, call them $C_{1}, \ldots, C_{N}$ with $N<M$ are just unions of disjoint subcollections of $N\left(x_{1}\right), \ldots, N\left(x_{M}\right)$. We have that for any $x, y \in C_{j}$,

$$
d_{c h, B}(x, y)<4 n M .
$$

We pause for a brief lemma which we will use to estimate the size of one of the $C_{j}$ 's.

Lemma 3.2. Let $A \subset \mathbb{R}^{n}$ be any measurable set of finite Lebesgue measure, then

$$
D_{A}=\frac{|A|^{n-1}}{\prod_{j=1}^{n}\left|\pi_{j}(A)\right|} \leq 1
$$

We refer to $D_{A}$ as the checkerboard density of $A$. The proof is simply to apply the 1-dimensional Hölder's inequality, $n$ times to

$$
\int \chi_{\pi_{1}(A)}\left(x_{2}, \ldots, x_{n}\right) \chi_{\pi_{2}(A)}\left(x_{1}, x_{3}, \ldots, x_{n}\right) \ldots \chi_{\pi_{n}(A)}\left(x_{1}, \ldots, x_{n-1}\right) .
$$

It is of some interest to note that the above argument also gives a proof of the Sobolev imbedding Theorem (cf. [GT, p. 156, equation $(7.27)])$. That this link should exist is natural because both the Sobolev imbedding Theorem and Theorem 1.3 concern giving global properties of functions in terms of their behavior on one dimensional slices.

Now we proceed to estimate the size of a $C_{j}$. We observe first that

$$
\sum_{j=1}^{N}\left|C_{j}\right|=\left|B_{n}\right| \geq\left(1-\frac{\delta}{2}\right)^{1 / n} \varepsilon\left|Q^{n}\right| .
$$

On the other hand, since the $C_{j}$ 's are checkerboard disjoint, their ( $n-$ 1)-dimensional projections are disjoint and hence we have for each $k \in$ $\{1, \ldots, n\}$,

$$
\sum_{j=1}^{N}\left|\pi_{k}\left(C_{j}\right)\right|=\left|\pi_{k}\left(B_{n}\right)\right|
$$


Applying Hölder's inequality yields

$$
\sum_{j=1}^{N} \prod_{k=1}^{n}\left|\pi_{k}\left(C_{j}\right)\right|^{1 / n} \leq \prod_{k=1}^{n}\left(\sum_{j=1}^{N}\left|\pi_{k}\left(C_{j}\right)\right|\right)^{1 / n} \leq \prod_{k=1}^{n}\left|\pi_{k}\left(B_{n}\right)\right|^{1 / n} .
$$

Hence, there is at least one $j$ for which

$$
\frac{\left|C_{j}\right|}{\prod_{k=1}^{n}\left|\pi_{k}\left(C_{j}\right)\right|^{1 / n}} \geq \frac{\left|B_{n}\right|}{\prod_{k=1}^{n}\left|\pi_{k}(B)\right|^{1 / n}}
$$

Taking the previous equation to the power $n$, we arrive at the main inequality

$$
\left|C_{j}\right| D_{C_{j}} \geq\left|B_{n}\right| D_{B_{n}}
$$

Observe that in particular,

$$
\left|B_{n}\right| D_{B_{n}} \geq\left(\frac{\left|B_{n}\right|}{\left|Q^{n}\right|}\right)^{n}\left|Q^{n}\right| \geq\left(1-\frac{\delta}{2}\right) \varepsilon^{n}
$$

Hence, since $D_{C_{j}} \leq 1$, one has that $\left|C_{j}\right| \geq(3 \varepsilon / 4)^{n}\left|Q^{n}\right|$. But what is more, we have a procedure for taking any subset $S$ of $B_{n}$ in any cube $Q^{\prime}$ and finding a subset $\tilde{S} \subset S$ which is checkerboard connected through $B$ with checkerboard diameter bounded by $8 M l\left(Q^{\prime}\right)$, so that

$$
|\tilde{S}| D_{\tilde{S}} \geq|S| D_{S}
$$

To see this, just dilate $Q^{\prime}$ into $[0,1]^{n}$ and follow the above argument. We require another lemma.

Lemma 3.3. Let $t_{1}, \ldots, t_{n-1}, s \in(0,1)$, then we have

$$
\frac{s^{n}}{t_{1} t_{2} \cdots t_{n-1}}+\frac{(1-s)^{n}}{\left(1-t_{1}\right)\left(1-t_{2}\right) \cdots\left(1-t_{n-1}\right)} \geq 1 \text {. }
$$

Proof. It is clear that the minimum of

$$
f(s)=\frac{s^{n}}{t_{1} t_{2} \cdots t_{n-1}}+\frac{(1-s)^{n}}{\left(1-t_{1}\right)\left(1-t_{2}\right) \cdots\left(1-t_{n-1}\right)},
$$


lies on the interior of $(0,1)$. Setting $f^{\prime}(s)=0$ gives

$$
s=\frac{\left(t_{1} t_{2} \cdots t_{n-1}\right)^{1 /(n-1)}}{\left(t_{1} t_{2} \cdots t_{n-1}\right)^{1 /(n-1)}+\left(\left(1-t_{1}\right)\left(1-t_{2}\right) \cdots\left(1-t_{n-1}\right)\right)^{1 /(n-1)}} .
$$

substituting back into $f$, gives that for any $s$,

$$
f(s) \geq\left(\frac{1}{\left(t_{1} t_{2} \cdots t_{n-1}\right)^{1 /(n-1)}+\left(\prod_{j=1}^{n-1}\left(1-t_{j}\right)\right)^{1 /(n-1)}}\right)^{n-1} .
$$

Now Jensen's inequality guarantees that $f(s) \geq 1$.

Now let $A_{0}$ be the set $C_{j}$ and define $f_{0}$ to be the constant function $D_{A_{0}}$ on $A_{0}$. Then the inequality ( $)$ may be rewritten as

$$
\int_{A_{0}} f_{0} \geq\left|B_{n}\right| D_{B_{n}}
$$

Then we obtain $A_{1}$ and $f_{1}$ as follows: We divide the cube $[0,1]^{n}$ into its dyadic children $Q_{1}, \ldots, Q_{2^{n}}$ and the set $A_{0}$ into $A_{0,1}, \ldots A_{0,2^{n}}$ with $A_{0, j}=A_{0} \cap Q_{j}$. Then Lemma 3.3 implies that

$$
\sum_{j=1}^{2^{n}}\left|A_{0, j}\right| D_{A_{0, j}} \geq \int_{A_{0}} f_{0}
$$

This is because when we chop a set $C$ into $C_{l}$ and $C_{r}$ one the left and right sides of a hyperplane $x_{j}=c$ then $\pi_{k}\left(C_{l}\right)$ is disjoint from $\pi_{k}\left(C_{r}\right)$ for $k \neq j$. We chop $A_{0}$ once in each coordinate direction to obtain (1).

Now for each nonempty $A_{0, j}$ we find $S_{j}$, a checkerboard connected component of $A_{0, j}$ in $Q_{j}$. As before, it will have the properties that,

$$
\left|S_{j}\right| D_{S_{j}} \geq\left|A_{0, j}\right| D_{A_{0, j}},
$$

and for any $x, y \in S_{j}$,

$$
d_{c h, B}(x, y) \leq 8 M n l\left(Q_{j}\right) .
$$

This last is true since $A_{0, j} \subset B_{n} \cap Q_{j}$ and any connected component of $B_{n} \cap Q_{j}$ which intersects $A_{0, j}$ is contained in $A_{0, j}$. Now we define 
$\cup S_{j}=A_{1} \subset A_{0}$ and we let $f_{1}$ be the function on $A_{1}$ which is constant on each $S_{j}$ and equals $D_{S_{j}}$. We have shown that

$$
\int_{A_{1}} f_{1} \geq \int_{A_{0}} f_{0}
$$

We proceed recursively producing $A_{j}$ from $A_{j-1}$ by letting the cubes at generation $j-1$ give birth, and letting $f_{j}$ be the function which is constant on the intersection of $A_{j}$ and cubes of the $j$-th generation and is equal there to the density of that intersection. Thus

$$
\int_{A_{j}} f_{j} \geq \int_{A_{j-1}} f_{j-1},
$$

and we have found a decreasing sequence of sets $A_{j}$ and a sequence of functions $f_{j}$ supported on $A_{j}$ and bounded by 1 so that for each $j$,

$$
\int_{A_{j}} f_{j} \geq\left|B_{n}\right| D_{B_{n}} .
$$

In particular, this implies that

$$
\left|A_{j}\right| \geq\left|B_{n}\right| D_{B_{n}}
$$

and hence

$$
\left|A_{\infty}\right| \geq\left|B_{n}\right| D_{B_{n}} \geq\left(1-\frac{\delta}{2}\right) \varepsilon^{n}
$$

where $A_{\infty}=\cap A_{n}$. We have in addition that for any $x, y \in A_{\infty}$,

$$
d_{c h, B}(x, y) \leq 8 M n d_{d}(x, y)
$$

where $d_{d}(x, y)$ is the dyadic distance between $x$ and $y$, i.e. the sidelength of the smallest dyadic cube containing both $x$ and $y$. The equations (2) and (3) are almost the statement of the theorem but for the appearance of dyadic distance instead of Euclidean distance. We must trim $A_{\infty}$ a little bit more in order to rectify this difficulty.

Now as we did to $B$, we define

$$
A_{\infty, 1}=\left\{x \in A_{\infty}: \mathcal{M}_{1}\left(\chi_{A_{\infty}^{c}}\right)(x)<1-\alpha \varepsilon^{n}\right\}
$$


and recursively for $j \leq n$,

$$
A_{\infty, j}=\left\{x \in A_{\infty, j-1}: \mathcal{M}_{j}\left(\chi_{A_{\infty, j-1}^{c}}\right)(x)<1-\alpha \varepsilon^{n}\right\} .
$$

By choosing $\alpha$ sufficiently small, we may arrange that $\left|A_{\infty, n}\right| \geq$ $(\varepsilon / 2)^{n}\left|Q^{n}\right|$. Let $D$ be the set of points in $\mathbb{R}^{n}$ one of whose coordinates is a dyadic rational. The set $D$ has measure 0 . We claim that $A_{\infty, n} \backslash D$ is the desired set $A$.

We shall refer to a cube's face of codimension 1 as walls. Every cube has $2 n$ walls which are naturally divided into $n$ pairs of opposite walls. Each such pair corresponds canonically to a coordinate direction $j$, namely the direction for which the coordinate function $x_{j}$ is constant on both faces in the pair. We say that two dyadic cubes $Q_{1}$ and $Q_{2}$ with the same sidelength are neighboring provided that the euclidean distance is 0 . If this is the case and $Q_{1}, Q_{2}$ are distinct, then $Q_{1}$ and $Q_{2}$ share a face $F$ of codimension $k$ where $1 \leq k \leq n$ and $k$ is an integer. Let $j_{1}>j_{2}>\cdots>j_{k}$ be the coordinate directions whose coordinate functions are constant on $F$. Then any two points $x \in Q_{1}$ and $y \in Q_{2}$ may be joined by a path which is piecewise linear with pieces in the coordinate directions $j_{1}, j_{2}, \ldots, j_{k}$ in that order and with corners in cubes $\tilde{Q}_{r}$ with $1 \leq r \leq k-1$ where each $\tilde{Q}_{r}$ neighbors $Q_{2}$. The cube $\tilde{Q}_{r}$ has a face in common with $Q_{2}$ of codimension $k-r$ which is associated to the directions $j_{r+1}, \ldots, j_{k}$.

For any $x$ and $y$ in $A$ either $d_{d}(x, y) \leq C /\left(\alpha \varepsilon^{n}\right) d(x, y)$ or there is a scale $l$ for which there are cubes $Q_{1}$ and $Q_{2}$ with $x \in Q_{1}$ and $y \in Q_{2}$ of sidelength $2^{-l} \leq 10 \sqrt{n} /\left(\alpha \varepsilon^{n}\right)|x-y|$ which are neighbors with a common face $F$ of codimension $j$ associated to the directions and $j_{1}>\cdots>j_{k}$ of the previous paragraph. These can be chosen so that the distance $d_{x, Q}$ from $x$ to the boundary of $Q_{1}$ satisfies $d_{x, Q} \geq 2 \alpha \varepsilon^{n} 2^{-l}$.

By the definition of $A_{\infty, n}$, we may find a point $x_{1} \in A_{\infty, n-j_{1}} \cap \tilde{Q}_{1}$ which can be connected to $x$ by a line in the direction $j_{1}$. We may proceed recursively choosing $x_{2}, \ldots, x_{r}, y_{1}$ with $x_{l} \in \tilde{Q}_{l} \cap A_{\infty, n-j_{l}}$ and $y_{1} \in Q_{2} \cap A_{\infty}$. But then

$$
\begin{aligned}
d_{c h, b}(x, y) & \leq(n+8 M n) 2^{-l} \\
& \leq C(\delta)\left(n+8 n^{2(n-1)} \varepsilon^{n-1}\right) n^{5 / 2} \sqrt{n} \varepsilon^{-n}|x-y| \\
& \leq C(\delta)\left(n^{3 / 2} \varepsilon^{-n}+\sqrt{n} n^{2 n} \varepsilon^{2 n-1}\right)|x-y| .
\end{aligned}
$$

This proves Theorem 1.3 and Corollary 1.4 


\section{Proof of Theorems 1.1 and Theorem 1.2.}

In this section, we apply the checkerboard Theorem and Proposition 2.1 to obtain a proof of Theorem 1.1. We observe that the proof of Theorem 1.2 is just a recursive iteration of the proof of Theorem 1.1.

Proof of theOREM 1.1. Let $e_{n}=(0,0, \ldots, 1)$ be the unit vector in the $n$-th coordinate direction and let $\pi_{n}$ be the projection of $\mathbb{R}^{n}$ into the hyperplane perpendicular to $e_{n}$. Let $Q$ be the cube in $\mathbb{R}^{n-1} \times\{0\}$ which is centered at the origin and which has sidelength $1 /(2 \sqrt{n-1})$. We will find a Lipschitz graph $\Gamma$ having large intersection with $S$ so that $\pi_{n}(\Gamma) \subset Q$.

For $v$ any unit vector in $\mathbb{R}^{n-1}$ let $v^{\perp}$ denote the $(n-2)$-plane of vectors perpendicular to $v$ in $\mathbb{R}^{n-1}$, let $P_{v}$ denote the 2 -plane in $\mathbb{R}^{n}$ spanned by $v$ and $e_{n}$, and for $w \in v^{\perp}$ denote by $P_{v, w}$ the translate $P_{v}+w$.

Consider $S \cap P_{v, w}$ for $w \in Q$. For $\lambda>0$, a real number, let

$$
B_{\lambda, v}=\left\{w \in v^{\perp}: \mathcal{H}^{1}\left(S \cap P_{v, w}\right)>\lambda\right\} .
$$

By the Slice Theorem ([Si, p. 156]) $\left|B_{\lambda, v}\right| \leq M / \lambda$. Further for each $w \in Q$, we have that $P_{v, w} \cap S$ separates 0 from $\infty$ in $P_{v, w}$. If we have further that $w \notin B_{\lambda, v}$, then we can apply Proposition 2.1 to a subset of $P_{v, w} \cap S$. This is because any connected rectifiable set with finite length can be parametrized. (see [DS]) We parametrize a component of the boundary of the domain containing $\infty$ in $P_{v, w} \backslash S$ which separates 0 from $\infty$. We apply Proposition 2.1 to this subset to obtain a 1dimension Lipschitz graph $\Gamma_{v, w, C}$ where $C$ is the Lipschitz constant in Proposition 2.1. Recall that $C$ depends only on the length of the curve and the choice of $\varepsilon$, which is at our disposal. Define

$$
\Gamma_{v, C, \lambda}=\bigcup_{w \in Q \backslash B_{\lambda}} \Gamma_{v, w, C}
$$

By choosing $C$ and $\lambda$ sufficiently large but depending only on $n$ and $M$, the measure of $S$, we may arrange that

$$
\left|\pi_{n}\left(\Gamma_{v, C, \lambda}\right) \cap Q\right| \geq|Q|-\varepsilon,
$$

for whichever $\varepsilon>0$ we wish.

We need a small Lemma. 
Lemma 4.1. Let $X$ be a measure space of total measure $M$. Fix $\varepsilon>0$ and $N \geq 2(M / \varepsilon)^{2}+1$. Let $A_{1}, \ldots, A_{N}$ be subsets of $X$ of measure $\geq \varepsilon$. Then there for $\varepsilon$ sufficiently small there exist $j, k$ with $\left|A_{j} \cap A_{k}\right| \geq c \varepsilon^{3} / M^{2}$ where $c \geq 0$ is a universal constant.

Proof. Choose $N_{0} \leq N=\left[(M / \varepsilon)^{2}+1\right]$ where $[x]$ denotes the greatest integer less than $x$. Let $\delta=c \varepsilon^{2} / M$ with $c$ to be specified later. Suppose the Lemma is false. Then $\left|A_{j} \backslash A_{1} \cup \cdots \cup A_{j-1}\right| \geq(\varepsilon-(j-1)) \delta$. Now

$$
|X|=M \geq \sum_{j}\left|A_{j} \backslash A_{1} \cup \cdots \cup A_{j-1}\right| \geq \delta \sum_{j=1}^{N_{0}} j \geq c \delta N_{0}^{2} \geq \frac{M^{2}}{\varepsilon} .
$$

But for $\varepsilon$ sufficiently small, this is a contradiction.

Hence we may find $v_{1}, \ldots, v_{n-1}$ linearly independent vectors in $\mathbb{R}^{n-1}$ with uniformly large angle between any pair so that

$$
\left|\pi_{n-1}\left(\bigcap \Gamma_{v_{j}, C, \lambda}\right) \cap Q\right| \geq \frac{1}{C M^{n-1}},
$$

and so that the smallest covering of $Q$ by parallelpipeds $P_{1}, P_{2}, \ldots, P_{K}$ with edges in the directions $v_{j}$ has $K$ depending only on $M$ and $n$. For some $k$ then, we have that with

$$
A_{k}=\pi_{n-1}\left(\bigcap \Gamma_{v_{j}, C, \lambda}\right) \cap P_{k}
$$

then $\left|A_{k}\right| \geq 1 /\left(10 K M^{N}\right)$, where $N>0$ depends only on $n$. To $A_{k}$, we apply the checkerboard theorem on $P_{k}$. This proves Theorem 1.1.

Proof of Theorem 1.2. Observe that in the proof of Theorem 1.1 above, we did not strongly use the fact that for some $\varepsilon>0$ of our choosing, we have that $\left|\Gamma_{v, C, \lambda}\right| \geq 1-\varepsilon$. If we had simply had that $\left|\Gamma_{v, C, \lambda}\right| \geq \delta /\left(10 n^{2}\right)$ for instance, we would have found a universally large intersection with universally bounded Lipschitz constant where these depend only on $\delta$ and $n$. Also, we do not have to use the cube $Q$ but may find a collection of $(n-1)$-cubes $Q_{1}, \ldots, Q_{5 n^{2}}$ so that the sectors over them cover the $(n-1)$-sphere, and define $\Gamma_{v, C, \lambda}$ for $v$ in any of these cubes. Then we choose $\varepsilon$ in Proposition 2.1 so that each $\Gamma_{v, C, \lambda}$ has projection onto $Q_{j}$ with measure at least $\left|Q_{j}\right|(1-\delta / 2)$. Then we use the proof of Theorem 1.1 to extract $\Gamma_{1}$ above the cube $Q_{1}$. We now 
replace the $\Gamma_{v, C, \lambda}$ 's by $\Gamma_{v, C, \lambda} \backslash \Gamma_{1}$ and we may continue recursively until for every cube $Q_{j}$ there is a $v \in Q_{j}$ so that $\Gamma_{v, C, \lambda} \backslash \Gamma_{1} \cup \Gamma_{l}$ has measure less than $\delta /\left(10 n^{2}\right)$. At this point, $\left|\pi\left(\Gamma_{1} \cup \cdots \cup \Gamma_{l}\right)\right| \geq \omega_{n}(1-\delta)$.

\section{Counterexample.}

Given two sets $E^{\prime} \subset E \subset[0,1]^{n}$, we define the (checkerboard) connectivity of $E^{\prime}$ through $E$,

$$
\gamma_{E}\left(E^{\prime}\right)=\sup _{x, y \in E^{\prime}} \frac{d_{c h, E}(x, y)}{d(x, y)},
$$

where $d(x, y)$ denotes the euclidean distance between $x$ and $y$.

Theorem 5.1. For every $\varepsilon<1 / 2$ there is a set $E \subset[0,1] \times[0,1]$, $|E| \geq \varepsilon / 2$, with the following property: for every $c>0$ there is $c^{\prime}>0$, depending only on $c$ and not on $E$ nor $E^{\prime}$ so that, if $E^{\prime} \subset E$ and $\left|E^{\prime}\right| \geq c \varepsilon^{2}$, then $\gamma_{E}\left(E^{\prime}\right)>c^{\prime}(\log (1 / \varepsilon))^{1 / 2}(\log \log (1 / \varepsilon))^{-1 / 2}$.

We pick $\varepsilon=1 / N^{2}, N$ being a natural number. Divide the unit square into $1 / \varepsilon$ disjoint subcubes,

$$
Q_{n, m}=[n \sqrt{\varepsilon},(n+1) \sqrt{\varepsilon}] \times[m \sqrt{\varepsilon},(m+1) \sqrt{\varepsilon}],
$$

for $n, m=0,1,2, \ldots, 1 / \sqrt{\varepsilon}-1$. To describe the set $E$ we define $E \cap Q_{n, m}$

$$
E \cap Q_{n, m}:=\bigcup_{k=0}^{1 / \varepsilon-1-n m} Q_{n, m}^{k}
$$

where

$$
\begin{aligned}
Q_{n, m}^{k}= & {\left[n \varepsilon^{1 / 2}+(k+n m) \varepsilon^{3 / 2}, n \varepsilon^{1 / 2}+(k+n m+1) \varepsilon^{3 / 2}\right] } \\
& \times\left[m \varepsilon^{1 / 2}+k \varepsilon^{3 / 2}, m \varepsilon^{1 / 2}+(k+1) \varepsilon^{3 / 2}\right] .
\end{aligned}
$$

It is easy to see that $|E| \geq \varepsilon / 2$.

Let us denote $\gamma=\gamma_{E}\left(E^{\prime}\right)$. We can assume that $Q_{n, m}^{i} \subset E^{\prime}$ whenever $Q_{n, m}^{i} \cap E^{\prime} \neq \varnothing$. 


\section{Lemma 5.2.}

a) Assume $Q_{n, m}^{i}, Q_{n+k, m+l}^{j} \subset E^{\prime}$. Then

$$
j-i=n l+O\left(\gamma^{2}(l+k+1)^{2}\right) .
$$

b) If $\gamma \leq 1 /(2 \sqrt{\varepsilon})$, then $\#\left\{k: Q_{n, m}^{k} \cap E^{\prime} \neq \varnothing\right\} \leq 1$ for all $n, m$.

PROOF. The authors would recommend to the reader to sketch a picture of $E$.

a) We may restrict our attention only to paths which pass the centers of small cubes $Q_{n, m}^{k}$ thus reducing everything to essentially a problem in graph theory. Every such path is composed of elementary steps - i.e. vertical or horizontal lines from a large cube $Q_{n, m}$ to one of its neighbors. An elementary step has length $\sim \sqrt{\varepsilon}$ and connects $Q_{n, m}^{k}$ to one of $Q_{n+1, m}^{k}, Q_{n-1, m}^{k}, Q_{n, m-1}^{k+n}$, or $Q_{n, m+1}^{k-n}$.

Now, by assumption, $Q_{n, m}^{i}$ and $Q_{n+k, m+l}^{j}$ are connected by a path composed of $M$ elementary steps with $M \leq \gamma(l+k+1)$. Then the cubes $Q_{n, m}^{i}$ and $Q_{n+k, m+l}^{j}$ are joined through a sequence $Q_{b(t), c(t)}^{a(t)}$ where $a, b, c$ are integer valued functions and $t$ runs from 1 to $M$. We always have $n-O(\gamma(l+k)) \leq b(t) \leq n+O(\gamma(l+k))$. Each upwards step increases $a(t)$ by $b(t)$ while each downwards one decreases it by $b(t)$. There must be $l$ more upwards steps than downwards ones and at most $M$ vertical steps. Thus $j-i=a(M)-a(0)=n l+O\left(\gamma^{2}(l+k+1)^{2}\right)$.

b) Assume false. Then there are $Q_{n, m}^{i}$ and $Q_{n, m}^{j} i \neq j$ joined by a path of consisting of less than or equal to $|i-j| \gamma \varepsilon$ elementary steps. This path must contain the same number of upward steps as downward steps. Thus by the argument above, one must have

$$
|i-j| \leq|i-j|^{2} \gamma^{2} \varepsilon^{2}
$$

but this is a contradiction since $|i-j| \leq 1 / \varepsilon$.

The following lemma tells us that given any cube $Q$ the set $E^{\prime}$ has to skip a considerable part of $Q$. The iterated application of this lemma will give us the bound on the measure of $E^{\prime}$.

Lemma 5.3. Given any cube $Q$ of sidelength $D \sqrt{\varepsilon}$, for some $D \geq 9 \gamma^{2}$, there is a subcube $Q^{\prime} \subset Q$ with sidelength $D \sqrt{\varepsilon} /\left(9 \gamma^{2}\right)$ so that $Q^{\prime} \cap E^{\prime}=$ $\varnothing$. 
Proof of the Lemma. Assume false. We divide $Q$ into $M^{2}=\left(9 \gamma^{2}\right)^{2}$ squares of sidelength $D \sqrt{\varepsilon} / M$. Our assumption means that there is a point of $E^{\prime}$ in each of them.

Without loss of generality, we assume that $Q=[0, D \sqrt{\varepsilon}]^{2}$. (We can do this by simply renumbering the cubes.) Denote $\tilde{Q}_{u, v}=[u D \sqrt{\varepsilon} / M$, $(u+1) D \sqrt{\varepsilon} / M] \times[v D \sqrt{\varepsilon} / M,(v+1) D \sqrt{\varepsilon} / M]$ and $x_{u, v} \in \tilde{Q}_{u, v} \cap E^{\prime}$. If $x_{u, v} \in Q_{n, m}^{l}$ denote also $l\left(x_{u, v}\right)=l, n\left(x_{u, v}\right)=n, m\left(x_{u, v}\right)=m$.

Then by the first part of Lemma 5.2

$$
\left|l\left(x_{0, v}\right)-l\left(x_{0, v-1}\right)\right| \leq \frac{D^{2}}{M^{2}}+O\left(\gamma^{2} \frac{D^{2}}{M^{2}}\right)=O\left(\gamma^{2} \frac{D^{2}}{M^{2}}\right)
$$

and

$$
l\left(x_{u-1,0}\right)-l\left(x_{u, 0}\right)=b_{u} u \frac{D}{M}+O\left(\gamma^{2} \frac{D^{2}}{M^{2}}\right)
$$

where the sequence $b_{u}=m\left(x_{u, 0}\right)-m\left(x_{u-1,0}\right)$ satisfies $\left|\sum_{r}^{s} b_{u}\right| \leq D / M$ for all $r<s$. (The inequality is obvious, since the sum telescopes and for any $u$, one has $\left.m\left(x_{u, 0}\right) \leq D / M\right)$.

Also,

$$
l\left(x_{u-1, M-1}\right)-l\left(x_{u, M-1}\right)=c_{u} u \frac{D}{M}+O\left(\gamma^{2} \frac{D^{2}}{M^{2}}\right),
$$

where $\left|\sum_{r}^{s} c_{u}\right| \leq D / M$ for all $r<s$.

From all these inequalities, we can get an estimate of

$$
\left|l\left(x_{M-1,0}\right)-l\left(x_{M-1, M-1}\right)\right|
$$

using the following

Fact 5.4. Let $\left\{d_{u}\right\}$ be a sequence so that $\left|\sum_{r}^{s} d_{u}\right| \leq D / M$ for all $r<s$. Then

$$
\left|\sum_{u=1}^{M} d_{u} u\right| \leq D
$$

Proof of FACT 5.4. One has simply

$$
\left|\sum_{u=1}^{M} u d_{u}\right|=\left|\sum_{l=1}^{M}\left(\sum_{u=l}^{M} d_{u}\right)\right| \leq \sum_{l=1}^{M} \frac{D}{M}=D .
$$


Now we obtain,

$$
\left|l\left(x_{M-1,0}\right)-l\left(x_{M-1, M-1}\right)\right| \leq O\left(\gamma^{2} \frac{D^{2}}{M}\right) .
$$

On the other hand, again by Lemma 5.2

$l\left(x_{M-1, v-1}\right)-l\left(x_{M-1, v}\right)=\left[m\left(x_{M-1, v-1}\right)-m\left(x_{M-1, v}\right)\right] D+O\left(\gamma^{2} \frac{D^{2}}{M}\right)$.

Hence,

$$
l\left(x_{M-1,0}\right)-l\left(x_{M-1, M-1}\right)=D^{2}+O\left(\gamma^{2} \frac{D^{2}}{M}\right) .
$$

Therefore $D^{2} \leq O\left(\gamma^{2} D^{2} / M\right)$, which is false if we take $M=c \gamma^{2}$, for a sufficiently big $c$.

REMARK 5.5. The argument of Lemma 5.3 actually proves that if $D \geq 18 \gamma^{2}$, then for every $k \in\left\{9 \gamma^{2}, 9 \gamma^{2}+1, \ldots, 18 \gamma^{2}\right\}$ there is a cube $\tilde{Q}_{u, v}$ with $\max (u, v)=k$ having empty intersection with $E^{\prime}$. Thus the measure of the union of those cubes is $|Q| /\left(36 \gamma^{2}\right)$.

Now, we are ready to end the computations. Starting with the unit cube $Q_{0}=[0,1] \times[0,1]$, we find a set $A_{1}$ which is a union of cubes of sidelength $1 /\left(18 \gamma^{2}\right)$ so that $E^{\prime} \subset A_{1}$ and $\left|A_{1}\right| \leq 1-1 /\left(18 \gamma^{2}\right)$. We take a grid of cubes of sidelength $1 /\left(18 \gamma^{2}\right)$. Applying the remark again to each of them, we find $A_{2} \subset A_{1}$, so that, $E^{\prime} \subset A_{2}, A_{2}$ is a union of cubes of sidelength $1 /\left(18 \gamma^{2}\right)^{2}$ and $\left|A_{2}\right| \leq\left(1-1 /\left(18 \gamma^{2}\right)\right)^{2}$. By induction, for any $m$ so that $1 /\left(18 \gamma^{2}\right)^{m} \geq \sqrt{\varepsilon}$, we find a subset $E^{\prime} \subset A_{m}$ union of cubes of sidelength $1 /\left(18 \gamma^{2}\right)^{m}$ with measure $\left|A_{m}\right| \leq\left(1-1 /\left(18 \gamma^{2}\right)\right)^{m}$.

Moreover, the second part of Lemma 5.2 implies that $\left|E^{\prime}\right| \leq \varepsilon^{2}\left|A_{m}\right|$ for all such $m$. Hence,

$$
\left|E^{\prime}\right| \leq \varepsilon^{2}\left(1-\frac{1}{18 \gamma^{2}}\right)^{\log (1 / \varepsilon) / \log \gamma}<\varepsilon^{2} e^{-d(\log (1 / \varepsilon) / \log \gamma) 1 / \gamma^{2}} \leq c \varepsilon^{2},
$$

when $\gamma^{2} \log \gamma \leq c^{\prime} \log (1 / \varepsilon)$.

\section{The Structure Theorem.}

The checkerboard theorem also provides a new approach to part of the "structure theorem" for $n$-dimensional $(n \in \mathbb{N})$ sets. To state that 
theorem in its "projection version" we need a definition. Here $G(m, n)$, $m \geq n$, denotes the Grassmannian manifold of all $n$-dimensional linear subspaces of $\mathbb{R}^{m}$, with its usual invariant measure.

Definition. For every plane $P \in G(m, n)$ denote by $\pi_{P}$ the orthogonal projection onto $P$. Given a set $E \subset \mathbb{R}^{m}$ we define the $n$-integral geometric measure of $E$ as

$$
\mathcal{U}_{n}(E)=\int_{G(m, n)} \mathcal{H}^{n}\left(\pi_{P}(E)\right) d P .
$$

Theorem 6.1 (The Structure Theorem). Let $E \subset \mathbb{R}^{m}, \mathcal{H}^{n}(E)<\infty$. $E$ has a decomposition into an $n$-rectifiable set $A$ and an $n$-unrectifiable set $E \backslash A$ and $\mathcal{U}_{n}(E \backslash A)=0$.

The following theorem is the special case of the structure theorem which we will show.

Theorem 6.2. Let $E \subset \mathbb{R}^{n+1}, 0<\mathcal{H}^{n}(E)<\infty$, so that $\mathcal{U}_{n}(E)>0$. Then there is $P \in G(m, n)$ and a Lipschitz graph $\mathcal{L}_{P}$ onto $P$ (i.e., there is a Lipschitz function $f_{P}: P \rightarrow \mathbb{R}^{m-n}$ whose graph is $\left.\mathcal{L}_{P}\right)$ such that $\mathcal{H}^{n}\left(E \cap \mathcal{L}_{P}\right)>0$.

Along with the density theorems, Theorem 6.1 is one of the central theorems in geometric measure theory. It was proven by Besicovitch [B] when $m=2, n=1$ and generalized by Federer [F]. The proof of Besicovitch's result can be found in any manual on that subject (see $[\mathrm{Fa}]$ ). The checkerboard Theorem allows us to deduce the theorem for higher dimensions, when $m=n+1$, from that case.

We will make use repeatedly of the following well known (and easy) fact

REMARK 6.3. Let $E$ be a $n$-rectifiable set in $\mathbb{R}^{n+k}$. Then for almost every $P \in G(n+k, n)$ there is a Lipschitz graph $\mathcal{L}_{P}$ onto $P$ so that $\mathcal{H}^{n}\left(E \cap \mathcal{L}_{P}\right)>0$.

Proof of Theorem 6.2. The proof will be by induction on $n$. The case $n=1$ is assumed to be known.

We will identify $G(l+1, l)$ and $S^{l}$ in the standard way: $P \in G(l+$ $1, l)$ is identified with its orthogonal vector $v \in S^{l}$. We will write $P=P_{v}$ and $\pi_{P_{v}}=\pi_{v}$. 
Assume that $E$ is a compact subset of $\mathbb{R}^{n+1}, \mathcal{H}^{n}(E)<\infty$ and $\mathcal{U}_{n}(E)>0$. Given $\omega \in S^{n}$ we define the family of planes orthogonal to $\omega, P_{\omega, t}=P_{\omega}+t \omega$. Denote $E_{\omega, t}=E \cap P_{\omega, t}$. Given any vector $\gamma$ orthogonal to $\omega$, (or, in other words $\omega \in P_{\gamma}$ ) we have

$$
\int_{\mathbb{R}} \mathcal{H}^{n-1}\left(\pi_{\gamma}\left(E_{\omega, t}\right)\right) d t=\mathcal{H}^{n}\left(\pi_{\gamma}(E)\right) .
$$

Consider the equator of the unit sphere

$$
S^{n-1}=\left\{x=\left(x_{1}, x_{2}, \ldots, x_{n+1}\right) \in S^{n}: x_{n+1}=0\right\} .
$$

Given $\theta \in S^{n-1}$ we define the meridian through $\theta$ as

$$
\mathcal{M}_{\theta}=\left\{x \in S^{n}: \pi(x)=t \theta, t>0\right\},
$$

where $\pi$ denotes the orthogonal projection onto the plane $\left\{x_{n}=0\right\}$.

The assumption on the integral geometric measure of $E$ implies that, for any $\theta \in S^{n-1}$,

$$
\begin{aligned}
\int_{\mathcal{M}_{\theta}} \int_{\mathbb{R}} \mathcal{U}_{n-1} & \left(E_{\omega, t}\right) d t d \omega \\
= & \int_{\mathcal{M}_{\theta}} \int_{\mathbb{R}} \int_{\gamma \in \omega^{\perp}} \mathcal{H}^{n-1}\left(\pi_{\gamma}\left(E_{\omega, t}\right)\right) d \mathcal{H}^{n-1}(\gamma) d t d \omega>0 .
\end{aligned}
$$

We now apply the $(n-1)$-dimensional theorem to $E_{\omega, t}$ whenever its integral geometric measure is positive. We obtain a set $C_{\theta} \subset \mathcal{M}_{\theta}$, $\mathcal{H}^{1}\left(C_{\theta}\right)>0$, such that, for all $\omega \in C_{\theta}$ there is $B(\omega) \subset\{P \subset G(n+$ $1, n), \omega \in P\} \approx\left\{v \in S^{n}: v\right.$ is orthogonal to $\left.\omega\right\}$, with $\mathcal{H}^{n-1}(B(\omega))>$ 0 , and for every $P \in B(\omega)$ there is a graph $\mathcal{L}_{P, \omega}$ over $P$, Lipschitz in the direction of $\omega, \mathcal{H}^{n}\left(\pi_{P}\left(E \cap \mathcal{L}_{P, \omega}\right)\right)>0$. In fact, for all $\omega \in C_{\theta}$, $\mathcal{H}^{n-1}(G(n+1, n) \cap\{\omega \in P\} \backslash B(\omega))=0$.

Let us denote

$$
\tilde{B}(\theta)=\bigcup\left\{B(\omega): \omega \in \mathcal{M}_{\theta}\right\}
$$

Then $\mathcal{H}^{n}(\tilde{B}(\theta))>0$. Therefore, using Fubini's theorem,

$$
\begin{aligned}
\int_{G(n+1, n)} \int_{S^{n-1}} & \chi_{\tilde{B}(\theta)}(P) d \mathcal{H}^{n-1}(\theta) d \mathcal{H}^{n}(P) \\
& =\int_{S^{n-1}} \int_{G(n+1, n)} \chi_{\tilde{B}(\theta)}(P) d \mathcal{H}^{n}(P) d \mathcal{H}^{n-1}(\theta)>0 .
\end{aligned}
$$


Hence, there is a plane $P$ such that $\mathcal{H}^{n-1}(\{\theta: P \in \tilde{B}(\theta)\})>0$. By the definition of $\tilde{B}(\theta)$ this is equivalent to $\mathcal{H}^{n-1}(\{\omega: P \in B(\omega)\})>0$. (Notice that $P \cap S^{n} \sim S^{n-1}$ ).

Let us denote $D(P)=\{\omega \in P: P \in B(\omega)\}$, and $E_{\omega}=E \cap \mathcal{L}_{P, \omega}$, $\omega \in D(P)$. Since $\mathcal{H}^{n-1}(D(P))>0, \mathcal{H}^{n}(E)<\infty$ and $\mathcal{H}^{n}\left(\pi_{P}\left(E_{\omega}\right)\right)>0$, for all $\omega \in D(P)$, then, we can find $\omega_{1}, \omega_{2}, \ldots, \omega_{n} \in D(P)$ linearly independent so that, $\mathcal{H}^{n}\left(\pi_{P}\left(\cap E_{\omega_{k}}\right)\right)>0$. Now, we apply the checkerboard theorem and conclude that there is $\tilde{E} \subset \cap E_{\omega_{k}}$ of positive $n$-dimensional measure and contained in a Lipschitz graph $\mathcal{L}_{P}$ over $P$.

\section{The tilt-excess Theorem.}

In this section, we discuss a special case of the checkerboard Theorem which immediately implies a version of the Almgren tilt-excess Theorem. For our purposes, the tilt-excess Theorem ( $c f .[\mathrm{A}])$ is the following.

Theorem 7.1. Let $\Omega$ be an open set and suppose the unit ball $B(0,1) \subset$ $\Omega$. Suppose further that

$$
\mathcal{H}^{n-1}(\partial \Omega) \leq(1+\varepsilon) \mathcal{H}^{n-1}\left(S^{n-1}\right)
$$

Then there exists a $C(n) \varepsilon^{1 / 3}$ Lipschitz graph $\Gamma$ over $S^{n-1}$ such that

$$
\mathcal{H}^{n-1}(\Gamma \triangle \partial \Omega) \leq \varepsilon^{1 / 3},
$$

where $\triangle$ denotes symmetric difference.

Theorem 7.1 will follow from the following simple version of the checkerboard theorem.

Lemma 7.2. Let $A \subset Q^{n}$ the unit cube in $\mathbb{R}^{n}$ with $|A|=1-\varepsilon$. Then for sufficiently small $\varepsilon$ (with small depending on $n$ ), there exists $B \subset A$ with $|B| \geq 1-C^{n} \varepsilon$ (with $C$ a universal constant) so that $B$ is $\sqrt{n}$ checkerboard connected through $A$.

Proof of Lemma 7.2. We define as in Section 3

$$
A_{1}=\left\{x \in A: \mathcal{M}_{1}\left(\chi_{B^{c}}\right)(x)<\frac{1}{4}\right\}
$$


and recursively for $j \leq n$,

$$
A_{j}=\left\{x \in B_{j-1}: \mathcal{M}_{j}\left(\chi_{B_{j-1}^{c}}\right)(x)<\frac{1}{4}\right\}
$$

Then we have by induction $\left|A_{n}\right| \geq 1-C^{n} \varepsilon$. We claim that $A_{n}$ is the desired set $B$.

Let $x=\left(\tilde{x}, x_{n}\right)$ and $y=\left(\tilde{y}, y_{n}\right)$. Consider the sets in $[0,1]$,

$$
S_{1}=\left\{t \in[0,1]:\left(\tilde{y}, x_{n}+t\left(y_{n}-x_{n}\right)\right) \in A_{n-1}\right\},
$$

and

$$
S_{2}=\left\{t \in[0,1]:\left(\tilde{x}, x_{n}+t\left(y_{n}-x_{n}\right)\right) \in A_{n-1}\right\} .
$$

Then by the definition of $A_{n}$ we have that $\left|S_{1}\right|,\left|S_{2}\right| \geq 3 / 4$. Hence, $\left|S_{1} \cap S_{2}\right| \geq 1 / 2$. Thus there exists $s \in S_{1} \cap S_{2}$. Thus letting $x^{n-1}=$ $(\tilde{x}, s)$ and $y^{n-1}=(\tilde{y}, s)$, we have $x^{n-1}, y^{n-1} \in A_{n-1}$. Analogously we define $x^{n-2}$ and $y^{n-2}$ by replacing $x$ and $y$ by $\tilde{x}$ and $\tilde{y}$. We proceed recursively, always having $x^{j}, y^{j} \in A_{j}$. Then the path from $x$ to $y$ through $x^{n-1}, \ldots, x^{j}, \ldots, y^{j}, \ldots, y^{n-1}$ has length at most $\sqrt{n}|x-y|$, and the lemma is proven.

Proof of Theorem 7.1. By Lemma 7.2 and the argument used to prove Theorem 1.1, there exists $\Gamma_{0}$ a $\sqrt{n}$ Lipschitz graph over $S^{n-1}$ such that

$$
\mathcal{H}^{n-1}\left(\Gamma_{0} \triangle \partial \Omega\right) \leq C(n) \varepsilon .
$$

Now let $\mu=\pi^{*}\left(\left.\mathcal{H}^{n-1}\right|_{\Gamma_{0}}\right)$ where $\pi$ is radial projection. Thus with $d \sigma$ defined as surface measure on $S^{n}$, we have that

$$
d \mu=(1+g) d \sigma
$$

with $\|g\|_{L^{1}\left(S^{n-1}\right)} \leq C(n) \varepsilon$. (Notice that if $\Gamma_{0}=\left\{\left(\theta, n(\theta) F_{0}(\theta)\right): \theta \in\right.$ $\left.S^{n-1}\right\}$ where $n(\theta)$ is the unit normal vector to $S^{n-1}$ at $\theta$, then we have that $g$ is on the order of $\left.|\nabla F|^{2}\right)$. Let $\mathcal{M}$ be the maximal operator on the sphere and let

$$
B=\left\{\mathcal{M} g>C(n) \varepsilon^{2 / 3}\right\},
$$

so that $\mathcal{H}^{n-1}\left(S^{n-1} \cap A\right)<\varepsilon^{1 / 3}$. Then we can replace $\Gamma_{0}$ by $\Gamma$ where, letting

$$
\Gamma=\left\{(\theta, n(\theta) F(\theta)): \theta \in S^{n-1}\right\},
$$


we have made $F$ differ from $F_{0}$ only on $B$. The reader may easily verify that one way of doing this is by observing that $B$ is open, and defining

$$
F(x)=\left(\phi_{d(x)} * F_{0}\right)
$$

for $x$ in $B$. Here $d(x)$ is the distance from $x$ to the boundary of $B$. We have fixed some positive bump function $\phi$ supported in the unit ball, and $\phi_{d(x)}$ is a version of it scaled to have support in the ball of radius $d(x)$. Thus the theorem is proved.

The reader may find it an amusing exercise to verify that the exponent $1 / 3$ is sharp.

\section{References.}

[A] Almgren, F., Approximation of Rectifiable Currents by Lipschitz Q valued functions. Seminar on Minimal Submanifolds. Ed. Enrico Bombieri, Princeton University Press, 1983.

[B] Besicovitch, A. S., On the fundamental geometric properties of linearly measurable plane sets of points (I). Math. Ann. 98 (1927), 422-464.

[DS] David, G., Semmes, S., Analysis on Uniformly Rectifiable Sets. American Mathematical Society, 1993.

[DS2] David, G., Semmes, S., Quasiminimal surfaces of codimension 1 and John domains. Preprint, Inst. Hautes Etudes Sci. Publ. Math. (1996).

[F] Federer, H., Geometric Measure Theory. Springer-Verlag, 1969.

[Fa] Falconer, K., The Geometry of Fractal Sets. Cambridge University Press, 1985.

[G] Guzman, M. de, Real Variable Methods in Fourier Analysis. North Holland, 1981.

[GT] Gilbart, D., Trudinger, N. S., Elliptic Partial Differential Equations of Second Order. Springer-Verlag, 1983.

[J] Jones, P. W., Lipschitz and bi-Lipschitz Functions. Revista Mat. Iberoamericana 4 (1988), 115-121.

[S] Semmes, S., Finding structure in sets with little smoothness. In: Proceeding of the International Congress of Mathematicians, Zürich (1994) 875-885. Birkhäuser-Verlag, 1995. 
[Si] Simon, L., Lectures on Geometric Measure Theory. Centre for Mathematical Analysis, ANU, 1984.

Recibido: 19 de octubre de 1.995

Peter W. Jones*

Department of Mathematics

10 Hillhouse Avenue

P. O. Box 208283

New Haven, CT 06520-8283

U.S.A.

jones@math. yale.edu
Nets Hawk Katz ${ }^{\dagger}$

Department of Mathematics The University of Edinburgh James Clerk Maxwell Building Room 5619 King's Buildings, Mayfield Road Edinburgh EH9 3JZ GREAT BRITAIN nets@maths .ed.ac.uk

and

Ana Vargas ${ }^{\ddagger}$

Departamento de Matemáticas Universidad Autónoma de Madrid 28049 Madrid ESPAÑA ana . vargas@uam. es

\footnotetext{
* The first author was supported by NSF grant DMS-92-13595.

$\dagger \quad$ The second author was partially supported by a National Science Foundation Postdoctoral Fellowship.

$\$$ The third author was partially supported by a Spanish DGICYT grant, PB94-149.
} 